\title{
Effects of Stratification and Hormone Treatments on Germination and Physio-Biochemical Properties of Taxus chinensis var. mairei Seed
}

\author{
Yanjie Zhang ${ }^{1}$, Shunbao Lu ${ }^{1}$, Handong Gao ${ }^{2 *}$ \\ ${ }^{1}$ College of Life Science, Jiangxi Normal University, Nanchang, China; ${ }^{2}$ College of Forest Resources and Environment, Nanjing \\ Forestry University, Nanjing, China. \\ Email: "gaohd@njfu.com.cn
}

Received April 27 $7^{\text {th }}, 2012$; revised May 25 ${ }^{\text {th }}, 2012$; accepted June $4^{\text {th }}, 2012$

\begin{abstract}
In order to learn dormancy mechanism of Taxtts chinensis var. mairei seed, effects of stratification and hormone treatments on physiological and biochemical indexes of the seeds after stratifying for eight months were researched. The content of amylase activity and soluble sugar gradually increased at first stratification, and then to the maximum for the end of after-ripening period. With the dormancy of seed relieving, some soluble sugar of endosperm was used or consumed by respiration and that led to their content decreased. Seed soluble protein was gradually accumulated at the beginning of stratification and then the initial raw material of protein synthesis of embryo during development had been used or in part converted into carbohydrates for energy consumption, so that the soluble protein content decreased. The fat and starch content slowly decreased during stratification, which showed that the storage of material had continually carrying out catabolism and conversion, which might provide energy sources for seed germination in the stratification process. The peroxidase activity of Taxus seeds increased gradually at the beginning of stratification, and then reached maximum point during stratification for 4 months in A1 and A2. The G-6-PDH and 6-PGDH joint activity was rapidly increased to reach the maximum during stratification for 4 months in A1, which showed the pentose phosphate pathway was activated at this time. The peroxidase activity rapidly increased might also be related to respiratory metabolic pathway during stratification. In plants, acid phosphatase hydrolyze PAM metabolites, namely phosphate material was related to energy metabolism, especially to high-energy phosphate, and phosphate enzyme activity changes affected the energy metabolism. The acid phosphatase activity of Taxus seeds in different treatments has no significant changes during stratification process, but there were two maximums. Energy metabolism might get benefit from the increase of acid phosphatase activity. There were significant differences in amylase activity, peroxidase activity, soluble sugar and soluble protein content with different treatments. Effect of promoting seed germination soaked by GA before stratification was obvious, but the concentration of GA should not be too high. The high concentration of GA caused seed to rot and reduce the seed germination rate. In this study, the highest germination rate of seed was $71.8 \%$ and soaked in 200 $\mathrm{mg} \cdot \mathrm{L}^{-1}$ gibberellin.
\end{abstract}

Keywords: Taxtts chinensis var. mairei Seed; Stratification; Hormone; Physiology and Biochemistry

\section{Introduction}

T. mairei is a rare Taxaceae Taxus species, and mainly distributed in China. T. mairei attracts universal attention for extracting significant anticancer activity of taxol in bark, twigs, leaves and other parts of plants [1-4]. However, the species have faced strong pressures of utilization in recent years and suffered a lot by devastating looting. T. mairei was listed as one of China's class key protected wild plants in 1999 [5]. Thus, biologists were seeking to increase the T. mairei population within a

\footnotetext{
"Corresponding author.
}

framework of forest resource management and genetic conservation. Seeds of T. mairei with a characteristic of morph-physiological deep dormancy have underdeveloped and dormant embryos, which must grow continuously to some length before seed dormancy could be broken. Its low natural reproduction results in very few endangered existing resources. Therefore, study on the life cycle of seed germination is important for biological characteristics of seeds of Taxus. Inclusions of seed germination and dormancy are suffered stratification and treatment of hormones, it is necessary to study physiological processes of seed germination and mechanism of 
germination in Taxus [6-8], for further study of understanding the occurring physiological and biochemical processes during stratification and treatment of hormones provided reference information of $T$. mairei seeds.

\section{Materials and Methods}

\subsection{Materials}

T. mairei fruits with the age 20 - 40 years that consist of scarlet or green cuplike arils were collected in the evergreen and deciduous broad-leaved mixed forest of 400 $500 \mathrm{~m}$ sea level in the valley and the slopes in Xiushui County, Jiujiang City, Jiangxi Province. The seeds were natural dried and then sealed into polyethylene bags and stored in the refrigerator. Experimental materials were fresh seeds. TGW was $65.048 \mathrm{~g}$.

\subsection{Methods}

Seeds were treated through five methods. A0 was airdried seeds at $25^{\circ} \mathrm{C}$ warm water immersion for $48 \mathrm{~h}$; $\mathrm{Al}$, A2 and A3 were soaked respectively by 200, 500, 1000 $\mathrm{mg} \cdot \mathrm{L}^{-1} \mathrm{GA} 3$ for $48 \mathrm{~h}$, respectively; A4 was washed in water for 1 week. Followed by all above treatments, the seeds were mixed with moist sand ratio to 1:3 stratification, first placed under a condition of variable temperature of $23^{\circ} \mathrm{C} / 10^{\circ} \mathrm{C}(12 \mathrm{~h}$ light $)$ for warm stratification for 4 months, and then placed in $5^{\circ} \mathrm{C}$ condition for cold stratification for 4 months; A5 was air-dried seeds that were soaked at $25^{\circ} \mathrm{C}$ in water for $48 \mathrm{~h}$, and mixed with moist sand and placed outdoors for natural stratification for 8 months. Seeds were sampled every one month at random during stratification, and placed into $-30^{\circ} \mathrm{C}$ refrigerator.

The contents of protein were measured by using the method of Commassie Blue G-250. Crude fat contents were measured by using method of Soxhlet extraction. The contents of soluble sugar and starch were measured by using the method of anthrone colorimetric determination [9].

Amylase Activity refers to experimental technique and the principles of Plant Physiology and Biochemistry [10]. Catalase activity was measured by using the method of guaiacol [11]. Acid phosphatase activity refers to seed biology study guide [12]. G-6-PDH and 6-PGDH activity was measured by using the method of co-determination.

Taxus seeds were randomly sampled from the treatments of A0, A1, A2, A3, A4 and A5 every 2 months for germination tests. One hundred seeds each treatment were collected with 4 replicates, and 100 seeds were placed in small germination boxes using sterilized sand as a substrate, and incubated at $25^{\circ} \mathrm{C}$ at incubator, ventilating and watering once 2 days during the period of 40 days after initial germination. The numbers of germination for seeds were recorded regularly.

\section{Results}

\subsection{Changes of Storage Substances in Seed}

Fat content of Taxus seeds was the most important storage substance in treatments in the early stratification and accounts for $60 \%$ (Figure 1(a)). Fat content in all treatments gradually decreased during stratification time. The rate of fat content decreased slowly until stratification for seven months and then decreased quickly in A0, A1 and A2, which illustrated fats slowly transfer into other substances and weaken metabolic activity. Degradation rate was slow in the stratification process that affected seed germination. ANOVA results showed that the difference of fat content between treatments was significant. Multiple comparisons showed that the difference was not significant among stratification from two to six months in $\mathrm{A} 0$, and from two to four months and five to six months in $\mathrm{A} 1$, and from one to two months, three to five months and five to seven months in A2, while the other differences among different stratification were significant.

The starch content in different treatments gradually decreased in general during stratification time (Figure 1(b)), while metabolic activity of seed and physiological and biochemical response increased. Demand of seeds for carbohydrates was increasing that led to starch content gradually decreased. The starch content in different stratification times in A2 was higher than that in other treatments at the same period, which showed the metabolic activity of seed in A2 was weaker than in other treatments. ANOVA showed that differences of the starch content in all treatments were significant. LSD showed that there was no significant difference of stratification from two to five months and six to seven months in $\mathrm{A} 0$, and from two to three months and four to five months in A1, and from one to two months in A2, and from one to three months and five to six months in A3, and from four to five months and four and six months in A5. The differences of starch content in other times were significant.

Changes of soluble sugar content in treatments were similar with protein content changes (Figure 1(c)). The difference of soluble sugar content was not significant during stratification for one month, while the soluble sugar content increased rapidly during various temperature stratification in $\mathrm{A} 2, \mathrm{~A} 3$ and $\mathrm{A} 5$, and all reached to the maximum during stratification for four months. The largest increase of soluble sugar content was $3.47 \%$ in A2. The soluble sugar content in A0 reaches to the maximum during stratification for three months. At the beginning of stratification, the increase of soluble sugar 

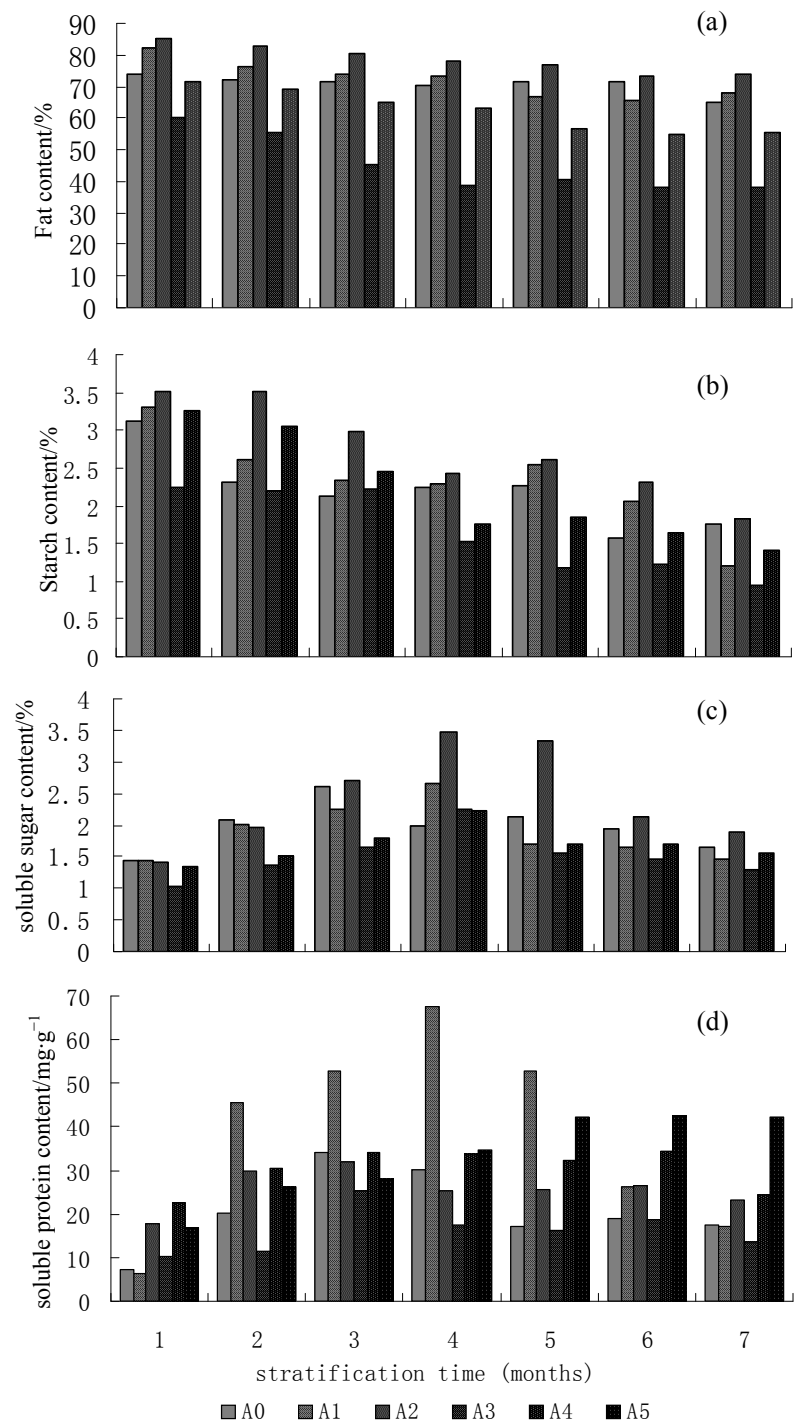

Figure 1. The storage substance content among different treatments in $T$. chinensis var. mairei seed.

content must come from crude fats, so the fat content gradually decreased, and then part of soluble sugar decreased due to the use of embryos or respiration of seeds. ANOVA results showed that the difference of soluble sugar content in all treatments was significant. Multiple comparisons showed that the difference of treatments during different stratification times is not significant.

Most of the soluble protein of plants was involved in various metabolic enzymes, and its content was an important indicator to understand the situation in plant metabolism. The soluble protein content generally increased and then decreased during stratification time (Figure 1(d)) in A0, A1, A2, A3 and A4. The biggest change was in treatment Alduring four months' stratification and was 10.8 times higher than the lowest, and then began to decrease, which showed that there was stronger meta- bolic activity of seeds in A1. The soluble protein content in A5 increased slowly. ANOVA showed that differences of protein content in treatments were significant. The multiple comparison showed that there was not significant difference from five to seven months in A0, and three and five months in A1, and two and three months, four and five months in A2, and one, two and seven months, and four to six months in $\mathrm{A} 3$, three and four months in A4, and six to seven months in A5, while differences in other times were significant.

\subsection{Changes of Key Enzyme Activity of Taxus Seed in Treatment}

The $\alpha$-amylase in Taxus seed had no obvious change in all treatments (Figure 2(a)). The $\alpha$-amylase activity in $\mathrm{A} 0, \mathrm{~A} 1$ and $\mathrm{A} 3$ increased gradually during variable temperature stratification for 3 months, and then decreased in treatment A0 and A1. The $\alpha$-amylase activity of treatment A3 increased rapidly during stratification for five months, and the maximum was $1.155 \mathrm{mg} \cdot \mathrm{g}^{-1} \cdot \mathrm{min}^{-1}$. The $\alpha$-amylase activity of treatment A2 reached the maximum during stratification for four months, and then decreased again. The maximum of $\alpha$-amylase activity gradually increased in treatments $\mathrm{A} 1, \mathrm{~A} 2$ and $\mathrm{A} 3$ with the increasing of exogenous concentration GA3 Enzymes. If the maximum counted as $100 \%$ in treatment $\mathrm{A} 0$, the maximum of $\alpha$-amylase activity in treatment $\mathrm{A} 1, \mathrm{~A} 2$ and A3 were $131.65 \%, 151.47 \%$ and $185.42 \%$ respectively, but the time of reaching to the maximum has been postponed, which showed that the effect was not affected by high concentration of exogenous GA3, and developed quick and ripened early of seeds treated by low concentrations of GA3 during stratification. ANOVA results showed that the differences of $\alpha$-amylase activity of every treatment were significant. The multiple comparisons showed that there were no significant differences of $\alpha$-amylase in treatments during the stratification.

The $\beta$-amylase activity gradually increased at the beginning of stratification (Figure 2(b)) and reached the maximum in $\mathrm{A} 0$ during stratification for 4 months, while in A1 during stratification for 2 months. The $\beta$-amylase activity reached to the first maximum in $\mathrm{A} 2$ and $\mathrm{A} 3$ during stratification for three and two months respectively, and then to the second maximum during stratification for 6 months. ANOVA showed that the differences of the $\beta$-amylase activity in treatments were significant. The multiple comparison showed that the difference was not significant during stratification for one and two months in $\mathrm{A} 0$, and for one and three months, and two and five months in A1, and one and four months, and four and seven months in A3. The differences in other treatments were significant during stratification times. 
The $(\alpha+\beta)$-amylase activity appeared two maximum in different treatments (Figure 2(c)). The $(\alpha+\beta)$-amylase activity reached maximum during stratification for three and six months in $\mathrm{A} 0$ and $\mathrm{A} 2$, and for three and seven months in A1, and for one and five months in A3. ANOVA results showed that the differences of $(\alpha+\beta)$ amylase activity were significant in every treatment. The multiple comparisons showed that the contents of $(\alpha+$ $\beta$ )-amylase activity have got no significant difference among treatments during stratification process. There was no significant difference of $(\alpha+\beta)$-amylase activity between months $1,2,5,7$ and months 4 and 6 in A0, between months 2, 3, 5, 7 and months 4 and 6 in A1, between months 1 and 5, months 2 and 4, and moths 1 and 7 in A2, between month 1 and months 3 and 7 in A3, while the differences in other times were significant.

Peroxidase was commonly in plant tissue and cells and participated in various physiological activities. The biochemical mechanism of seed dormancy proposed by Roberts mentioned that the proportion of pentose phosphate (PPP) approach was continuously strengthened during the whole respiration process and was an important condition determining germination of dormant seeds (Roberts E H. 1973). The peroxidase was closely associated with the PPP approach, and its activity could promote the PPP approach to enhance favorable conditions for the relieved seed dormancy. The peroxidase activity first increased and then decreased in all treatments during stratification (Figure 2(d)), while the stratification times reaching to the maximum were different in every treatment. The maximum times in A1 and A2 were stratification for four months, and for five months in $\mathrm{A} 0$ and $\mathrm{A} 4$, and for six months in A3 and A6. ANOVA results showed that the differences of peroxidase activity were significant in every treatment. The multiple comparisons showed that the contents of peroxidase activity have no significant differences among treatments during stratification time. There were no significant differences of peroxidase activity between months 1 and 2 in A0 and A4, between months 2 , and months 7 in A2, between months 3 and moths 7 in A3, between month 3 and months 4 in A5. Except these, the others were significant differences.

Acid phosphatase was a kind of key enzyme of hydrolysis organic phosphate in organism and in various seeds. The acid phosphatase activity reached maximum during stratification for one month in $\mathrm{A} 0$ and $\mathrm{A} 3$, and then appeared curve with "low-high-low" (Figure 2(e)). The acid phosphatase activity gradually increased during stratification, and reached the first maximum of 4.910 $\mathrm{nmol} \cdot \mathrm{mg}^{-1} \cdot \mathrm{min}^{-1}$ during stratification for four months, and the second maximum of $7.011 \mathrm{nmol} \cdot \mathrm{mg}^{-1} \cdot \mathrm{min}^{-1}$ in A2. There were two maximums in other treatments, but
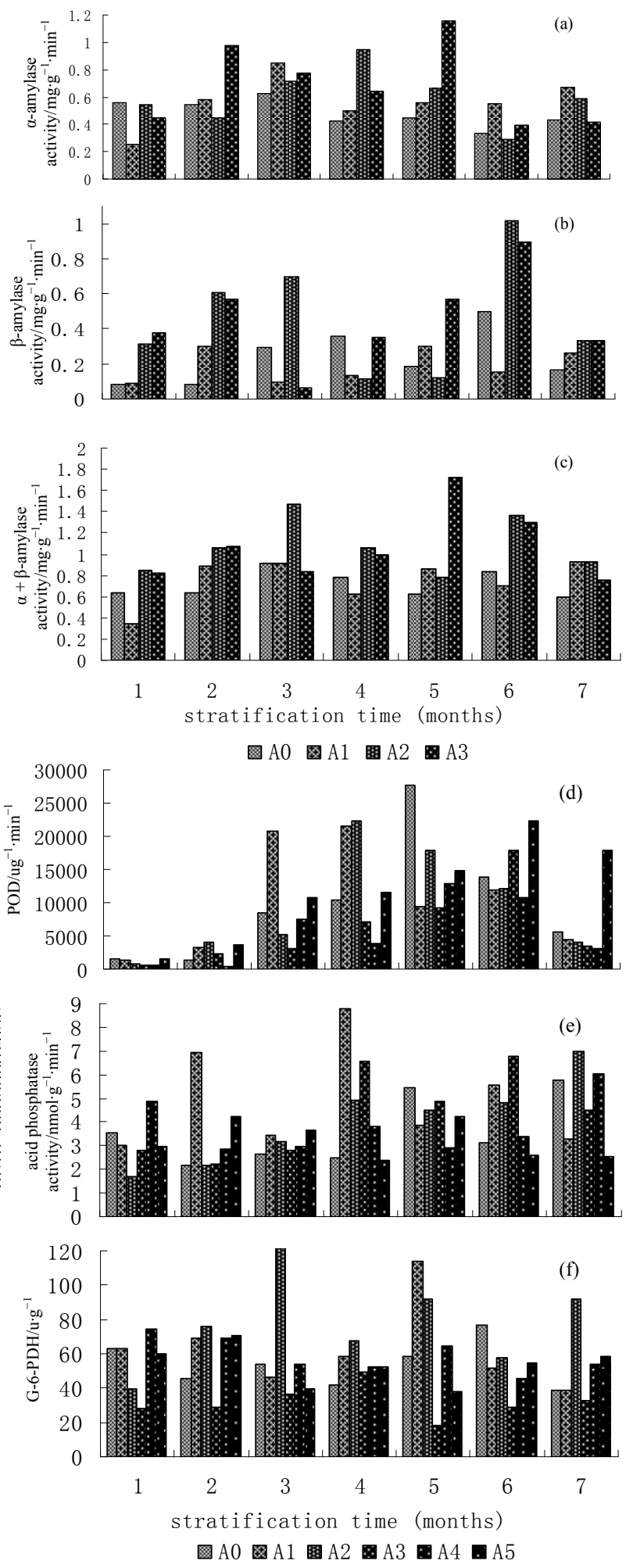

Figure 2. The enzyme activity among different treatments in T. chinensis var. mairei seed.

the stratification time was different. The maximum was in stratification for five and seven months in $\mathrm{A} 0$, and for 
two and four months in A1, and for four and six months in $\mathrm{A} 3$, and for one and seven months in A5, and for two and five months in treatment A5. ANOVA results showed that the acid phosphatase activity differences were significant in every treatment. The multiple comparisons showed that the contents of acid phosphatase activity have no significant difference among treatments during stratification. There were no significant differences in acid phosphatase activity between months 2, 3, 4 and months 4 and 7 and months 4 and 6 in A0, between months 1, 5, 7 and months 3, 5, 7 in A1, between months 2, 4, 6 in A2, between month 1 and 3 and months 4 and 6 and months 5 and 7 in A3, between month 2, 3, 5 in A4, between month 1 and 6 and months 2 and 5 and months 4 , 6, 7 in A5, while the other differences were significant.

Glucose-6-phosphate dehydrogenase (G-6-PDH) and 6-phosphogluconate dehydrogenase (6-PGDH) were the key restricting enzymes in the pentose phosphate pathway (PPP), and which reflected state of phosphate Pentose process. The change of enzyme activity was not obvious in different treatments (Figure 2(f)). The enzyme activity gradually decreased during stratification in $\mathrm{A} 0$, $\mathrm{A} 4$ and A5. The enzyme activity was $113.947 \mathrm{u} \cdot \mathrm{g}^{-1} \cdot \mathrm{min}^{-1}$ during stratification for five months in A1. The enzyme activity reached two maximums in the whole stratification process, respectively during stratification for three and five months, and then appeared curve by low-highlow-high in A3. ANOVA results showed that differences of the enzyme activity were significant in every treatment except stratification for four and seven months in A0. The multiple comparisons showed that the enzyme activity has no significant difference among treatments during stratification time. There were no significant differences of the enzyme activity between 1 and 4 months in A1, between 5 and 7 months in A2, between 1, 2 and 6 months in A3, between 3, 4 and 7 months in A4, between 1 and 7 months and months 3 and 5 and months 4 and 6 in $\mathrm{A} 5$, while the other differences were significant.

\subsection{Germination Rate of Taxus Seed}

These seeds did not germinate after warm stratification for four months (Table 1), but appeared some gap during stratification for 3 months. Only a few seeds germinated in A0, A1, A2, A3 and A4 after stratification for 6 months. The highest germination rate is only $8.0 \%$ in $\mathrm{A} 3$. The germination rate significantly increased after warm stratification for 8 months among all treatments, and the best effect of relieving dormancy was treatment A1, whose germination reached $71.8 \%$. The followed germination rate was $61.5 \%$ in $\mathrm{A} 4$. The germination rate was higher in A1, A2 and A4 than that in A0, which showed that germination rate of seed was obviously affected by Gibberellin soaking or water leaching. However, the germination rate was low in A3 when soaking in 1000 $\mathrm{mg} \cdot \mathrm{L}^{-1}$ gibberellin and seeds were found rot in high concentrations of gibberellic. There was no germination in the stratification process in A5. The multiple comparisons showed that $\mathrm{A} 1$ has the highest germination rate and there were significant differences among other treatments. The germination rate was high in A4 and there were significant differences among other treatments. The germination rate has no significant difference among treatment $\mathrm{A} 0$ and $\mathrm{A} 2$.

\section{Conclusions}

\subsection{Changes of Storage Material Content}

Seed germination process requires materials and energy to maintain living activities. These nutrients come mainly from storage substances in seeds such as the transformation and utilization of protein, starch and fat. The degradation of macromolecular storage substances transferred into variety soluble substances for embryo metabolism and growth process to use in stratification process. The changes of main storage substances showed that the embryo of mature seeds has not yet fully developed, and the embryo had continuous development and differentiation

Table 1. Germination percentage among different treatments of stratification during different periods T. chinensis var. mairei seed.

\begin{tabular}{cccccccc}
\hline period/m & A0 & A1 & A2 & A3 & A4 & A5 \\
\hline 0 & $0 \mathrm{Aa}$ & $0 \mathrm{Aa}$ & $0 \mathrm{Aa}$ & $0 \mathrm{Aa}$ & $0 \mathrm{Aa}$ & $0 \mathrm{Aa}$ & $0 \mathrm{Aa}$ \\
2 & $0 \mathrm{Aa}$ & $0 \mathrm{Aa}$ & $0 \mathrm{Aa}$ & $0 \mathrm{Aa}$ & $0 \mathrm{Aa}$ & $0 \mathrm{Aa}$ & $0 \mathrm{Aa}$ \\
4 & $0 \mathrm{Aa}$ & $0 \mathrm{Aa}$ & $0 \mathrm{Aa}$ & $0.6 \pm 1.2 \mathrm{Cb}$ & $0.1 .4 \mathrm{Dc}$ & $3.6 \pm 3.5 \mathrm{Dd}$ & $0 \mathrm{Aa}$ \\
\hline
\end{tabular}

Note: capital letters $\alpha=0.05$ of multiple comparisons, lowercase letters $\alpha=0.01$ of multiple comparisons. The difference is no significant in the same letters and significant in the different letters of the same column. $\mathrm{P}^{* *}<0.01$. 
in stratification process. In stratification process, the content of amylase activity and soluble sugar gradually increased at first, and then to the maximum for the end of after-ripening period. With the dormancy of seed relieving, some soluble sugar of endosperm was used or consumed by respiration and that led to their content decreased. Seed soluble protein was gradually accumulated at the beginning of stratification and then the initial raw material of protein synthesis of embryo during development had been used or in part converted into carbohydrates for energy consumption, so that the soluble protein content decreased. The fat and starch content slowly decreased during stratification, which showed that the storage of material had continually carrying out catabolism and conversion, which might provide energy sources for seed germination in the stratification process.

\subsection{Changes of Enzyme Activity}

The physiological mechanism of peroxidase in plant growth and development was unclear. PP hypothesis of Roberts and Tao et al. proposed that seed germination and dormancy were related to peroxidase and regulated by respiratory pathway in the EMP-TCA and the balance of PP $[13,14]$. The peroxidase activity of Taxus seeds increased gradually at the beginning of stratification, and then reached maximum point during stratification for 4 months in A1 and A2. The G-6-PDH and 6-PGDH joint activity was rapidly increased to reach the maximum during stratification for 4 months in A1, which showed the pentose phosphate pathway was activated at this time. The peroxidase activity rapidly increased might also be related to respiratory metabolic pathway during stratification. In plants, acid phosphatase hydrolyze PAM metabolites, namely phosphate material was related to energy metabolism, especially to high-energy phosphate, and phosphate enzyme activity changes affected the energy metabolism. The acid phosphatase activity of Taxus seeds in different treatments has no significant changes during stratification process, but there were two maximums. Energy metabolism might get benefit from the increase of acid phosphatase activity.

\subsection{Germination Rates of Seed in Different Treatment}

Effect of promoting seed germination soaked by GA before stratification was obvious, but the concentration of GA should not be too high. The high concentration of GA caused seed rot and reduce the germination rate. In this study, the highest germination rate of seed was $71.8 \%$ and soaked in $200 \mathrm{mg} \cdot \mathrm{L}^{-1}$ gibberellin, then followed by $61.5 \%$ by water leaching, and both of them were significantly higher than other treatments.

\section{Acknowledgements}

The work reported here has been funded by Projects from Ministry Education Youth Foundation of Jiangxi Province of China (GJJ11069) and Jiangxi Province Natural Science Foundation of China (20114BAB214007).

\section{REFERENCES}

[1] Y. J. Yuan, C. Li, Z. D. Hu, J. C. Wu and A. P. Zeng, "Fungal Elicitor-Induced Cell Apoptosis in Suspension Cultures of Taxus chinensis vat. mairei for Taxol Production," Process Biochemistry, Vol. 38, No. 2, 2002, pp. 193-198. doi:10.1016/S0032-9592(02)00071-7

[2] Y. J. Yuan, C. Li, J. C. Wu and Z. D. Hu, "A Model for Signal Transduction in Suspension Cultures of Taxus chinensis var. mairei Induced by an Oligosaccharide from Fusarium oxysporum," Biotechnology Letters, Vol. 24, No. 5, 2002, pp.407-412. doi:10.1023/A:1014598122300

[3] Y. J. Yuan, C. Li, Z. D. Hu and J. C. Wu, "A Double Oxidative Burst for Taxol Production in Suspension Cultures of Taxus chinensis var. mairei Induced by Oligosaccharide from Fusarium oxysprum," Enzyme and Microbial Technology, Vol. 30, No. 6, 2002, pp. 774-778. doi:10.1016/S0141-0229(02)00057-1

[4] C. Li, Y. J. Yuan, J. C. Wu and Z. D. Hu, "A Structured Kinetic Model for Suspension of Taxus chinensis var. mairei Induced by an Oligosaccharide from Frusarium oxysporum," Biotechnology Letters, Vol. 25, No. 16, 2003, pp. 1335-1343. doi:10.1023/A:1024980420790

[5] J. W. Cao and X. Y. Chen, "Research and Application on Taxus chinensis var. mairei Forest Technique," Forest Science and Technology Exploitation, Vol. 13, No. 5, 1999, pp. 12-13.

[6] W. Y. Yang and H. Guan, "Research Advance of Seed Germination Physiology," Seed, No. 5, 2002, pp. 31-32.

[7] H. Tang, W. Ning, X. L. Lu and Q. Y. Pan, "Effects of Hormone Treatment on Seed Storage Matter of Schisandra chinensen (Turcz.) Baill. during Low Temperature Stratification," Henan Agricultural Sciences, No. 6, 2008, pp. 92-94.

[8] X. L. Yang, S. H. Guo, J. W. Zhang, Y. F. Liu, C. Wang, Z. Li and H. F. Wang, "Effects of Stratification and Hormone Treatments on Physiological and Biochemical Indexes of Hawthorn Seed," Economic Forest Research, Vol. 27, No. 1, 2009, pp. 76-79.

[9] X. L. Huang and R. Z. Chen, "Seed Physiological Experiment Manual," China Agriculture Press, Beijing, 1998.

[10] J. Y. Wang, "Technology and Principle of Plant Physiology and Biochemical Experiments," Press of Northeast Forestry University, 2003.

[11] H. S. Li, "Physiology and Biochemistry of Plant Experimental Principle and Technical," Higher Education Press, 2006.

[12] S. Q. Song, "Seed Biology Research Guide," Science Press, Beijing, 2005. 
Properties of Taxus chinensis var. mairei Seed

[13] K. I. Tao and A. A. Khan, "Changes in Isoperonidases during Cold Treatment of Dormant Pear Embryo," Plant Physiology, Vol. 57, No. 1, 1976, pp. 1-4. doi:10.1104/pp.57.1.1
[14] E. H. Roberts, "Oxidative Processes and the Control of Seed Germination,” In: W. Heydecker, Ed., Seed Ecology, Butterworth, London, 1973, pp. 189-218. 